\title{
IT/Business Alignment als Managementherausforderung
}

Die AUtoren
Robert Winter
Karl Landert
Prof. Dr. Robert Winter
Universität St. Gallen
Institut für Wirtschaftsinformatik
Müller-Friedberg-Strasse 8
9000 St. Gallen
Schweiz
Robert.Winter@unisg.ch
http://www.iwi.unisg.ch
Karl Landert
Credit Suisse
ClO Private Banking Division \& CIO Europe
Uetlibergstrasse 231
8070 Zürich
Schweiz
http://www.credit-suisse.com

Die Organisationsgestaltung und die Gestaltung von Anwendungssystemen sind auf komplexe Weise miteinander verknüpft: Einerseits dienen Anwendungssysteme der Unterstützung betrieblicher Prozesse, d. h. ihre Gestaltung ist nach fachlichen Anforderungen auszurichten. Andererseits muss die Organisationsgestaltung die Geschäftspotenziale realisieren, die IT-Innovationen ermöglichen. Unter IT/Business Alignment wird die wechselseitige Abstimmung von Zielen, Strategien, Architekturen, Leistungen und Prozessen zwischen Informatikbereichen und Fachbereichen in Unternehmen verstanden. Im übertragenen Sinne ist IT/Business Alignment auch Grundlage einer effizienten Zusammenarbeit von Unternehmen (als Nachfrager von IT-Leistungen) und IT-Dienstleistern.

Gemäß einer Untersuchung von Luftman in MISQ Executive 2/2005 ist IT/Business Alignment das wichtigste Thema für ITManager. Zwar beschränkt sich die empirische Basis dieser Untersuchung auf 182 Unternehmen in den USA und die Jahre 2003-2004. Da aber IT/Business Alignment über alle Managementebenen, Branchen und Erfahrungsklassen hinweg konsistent Priorität 1 hat, darf davon ausgegangen werden, dass dies auch für Unternehmen ausserhalb der USA nicht völlig anders ist.

Die Wirtschaftsinformatik sollte deshalb geeignete Konzepte bereitstellen, um IT/ Business Alignment zu unterstützen. Dieses Schwerpunktheft umfasst vier Schwerpunktaufsätze, die in drei Begutachtungsund Auswahlzyklen aus ursprünglich 23 Einreichungen ausgewählt wurden:

- Der Beitrag von Baumöl präsentiert einen Ansatz zur situativen Methodenkonstruktion in Veränderungsprojekten. $\mathrm{Da}$ Alignment angesichts unterschiedlicher Lebenszyklen von fachlichen Artefakten und IT-Artefakten in Form von Gestaltungs- und Evolutionsprojekten umgesetzt wird, sind situative Methoden eine wichtige Voraussetzung für ein systematisches Vorgehen.

- Beukers et al. stellen ein Ansatz vor, der für die Domäne „Beschaffung“ verschiedene fachliche und IT-Perspektiven verbindet. Auf dieser Grundlage können nicht nur aufeinander abgestimmte Konzepte geschaffen werden, sondern es kann auch der realisierte AlignmentGrad gemessen und systematisch weiterentwickelt werden.

- Im Beitrag von Beimborn et al. wird im Rahmen einer empirischen Studie mit 1.000 deutschen Banken gezeigt, wie im Zusammenspiel von Fach- und IT-Ressourcen und dem Alignment beider
Bereiche die Geschäftsprozessleistung positiv beeinflusst werden kann. Die Untersuchung zeigt, dass sowohl ITRessourcen wie auch fachliche Ressourcen einen positiven Einfluss auf die Prozessleistung aufweisen, dass Alignment als eigenständiger Faktor einen Wertbeitrag aufweist und dass auch eine sehr gute IT Schwächen auf Seiten der Fachabteilungen nicht kompensieren kann.

- Kashanchi und Toland untersuchen, wie mit Hilfe von ITIL Alignment zwischen fachlichen Zielen und IT-Zielen erreicht werden kann. Die Grundlage dafür bildet das Strategic Alignment Model von Henderson and Venkatraman, in dem ITIL an die Stelle von „IS infrastructure and processes" tritt. Die konzeptionell gestützte Argumentation, dass ITIL Alignment unterstützt, wird durch Interviews untermauert.

Die vier Schwerpunktaufsätze werden ergänzt durch Beiträge der Rubriken „WISchlagwort" und „WI - Für Sie gesurft", die ebenfalls IT/Business Alignment adressieren. Das Interview mit den Herren Prof. Dr. Henning Kagermann und Prof. Dr. Hubert Österle zu ihrem neuen Buch „Geschäftsmodelle 2010“ beleuchtet das komplexe $\mathrm{Zu}$ sammenspiel von Fachlichkeit und IT aus der Perspektive des Top-Managements.

Unser Dank für den Erfolg dieses Schwerpunkthefts gebührt den 23 Autorenteams (davon fünf aus dem nicht deutschsprachigen Raum), die Ihre Arbeiten eingereicht haben. 25 Gutachterinnen und Gutachter haben die Beiträge bewertet und durch Ihre Verbesserungsvorschläge weiterentwickelt. Frau Anke Gericke, wissenschaftliche Mitarbeiterin am IWI-HSG, unterstützte die Herausgeber nicht nur effektiv und effizient, sondern leistete auch wertvolle inhaltliche Inputs.

Prof. Dr. Robert Winter Karl Landert 